\title{
Structural Analysis of Caulobacter crescentus and its Bacteriophage
}

\author{
R. Guerrero-Ferreira*, G. M. Williams*, J. M. Holl, and E. R. Wright**** \\ * Emory University School of Medicine, Division of Pediatric Infectious Diseases, Children's \\ Healthcare of Atlanta, 2015 Uppergate Drive NE, Atlanta, GA 30322 \\ ** Robert P. Apkarian Integrated Electron Microscopy Core, Emory University, 1521 Dickey \\ Drive, Atlanta, GA 30322
}

Caulobacter crescentus is a fresh-water, Gram-negative bacterium with a dimorphic life cycle [1]. A number of flagellotropic bacteriophages that infect members of Caulobacteraceae $(\alpha-$ proteobacteria) have been described [2]. C. crescentus, while in its swarmer state, or flagellated form, has been shown to be host to several flagellotropic bacteriophages, including the siphophages $\varphi \mathrm{Cb} 13$ and $\varphi \mathrm{CbK}[3]$. Two- and three-dimensional cryo-electron microscopy, together with adsorption kinetics assays of $\varphi \mathrm{Cb} 13$ and $\varphi \mathrm{CbK}$ phage-infected, wild type and mutant strains of $C$. crescentus (Table 1) were used to provide insight into the mechanisms of infection.

Our data demonstrate that $\varphi \mathrm{Cb} 13$ and $\varphi \mathrm{CbK}$ actively interact with the flagellum and are able to simultaneously attach to bacteriophage receptors on the cell pole. Here, we present evidence that the first interaction of the phage with the bacterial flagellum takes place through a filament on the phage head (Fig. 1). This contact with the flagellum facilitates aggregation of phage particles around the receptor (i.e. the pilus portals) on the bacterial cell surface, thereby increasing the likelihood of infection. Our cryo-EM analyses and infectivity assays demonstrate that cells with altered flagellar formation or function directly impact the success of phage adsorption and infection (Table 2).

It is possible that phage head filaments systematically underlie the initial interactions of phages with their hosts in other systems and represent an unprecedented mechanism of efficient phage propagation. Further studies will focus on the head filament of $\varphi \mathrm{Cb} 13$ and $\varphi \mathrm{CbK}$ to reveal its genetic origin, composition, and contribution to phage adaptation.

References

[1] J.S. Poindexter, Bacteriol. Rev. 28 (1964) 231.

[2] J.M. Schmidt and R.Y. Stanier, J. Gen. Microbiol. 39 (1965) 95.

[3] J.M. Schmidt, J. Gen. Microbiol. 45 (1966) 347.

[4] This research was supported by Emory University, Children's Healthcare of Atlanta, and the Georgia Research Alliance. Cryo-ET data was collected at the Emory University Robert P. Apkarian Integrated Electron Microscopy Core. We would like to thank Dr. Jeanne Poindexter, Dr. Patrick Viollier and, Dr. Bert Ely for providing bacterial strains. 
TABLE 1. Bacterial strains utilized during the course of this study.

\begin{tabular}{ll}
\hline Strain name & Phenotype \\
\hline C. crescentus NA1000 & Synchronizable derivative of CB15 \\
C. crescentus motA:Tn & Incomplete stator and deficient motility \\
C. crescentus cheB $144: \because T n$ & Counterclockwise flagellar rotation (CCW) \\
C. crescentus cheB $148:: T n$ & Counterclockwise flagellar rotation (CCW) \\
C. crescentus cheR $:: T n$ (NS209) & Clockwise flagellar rotation (CW) \\
C. crescentus che $::$ Tn (NS338) & Clockwise flagellar rotation (CW) \\
C. crescentus $\triangle$ tipF & Flagellum not present. Non-swarmer cells \\
C. crescentus $\triangle$ pilA & Pili not present \\
\hline
\end{tabular}

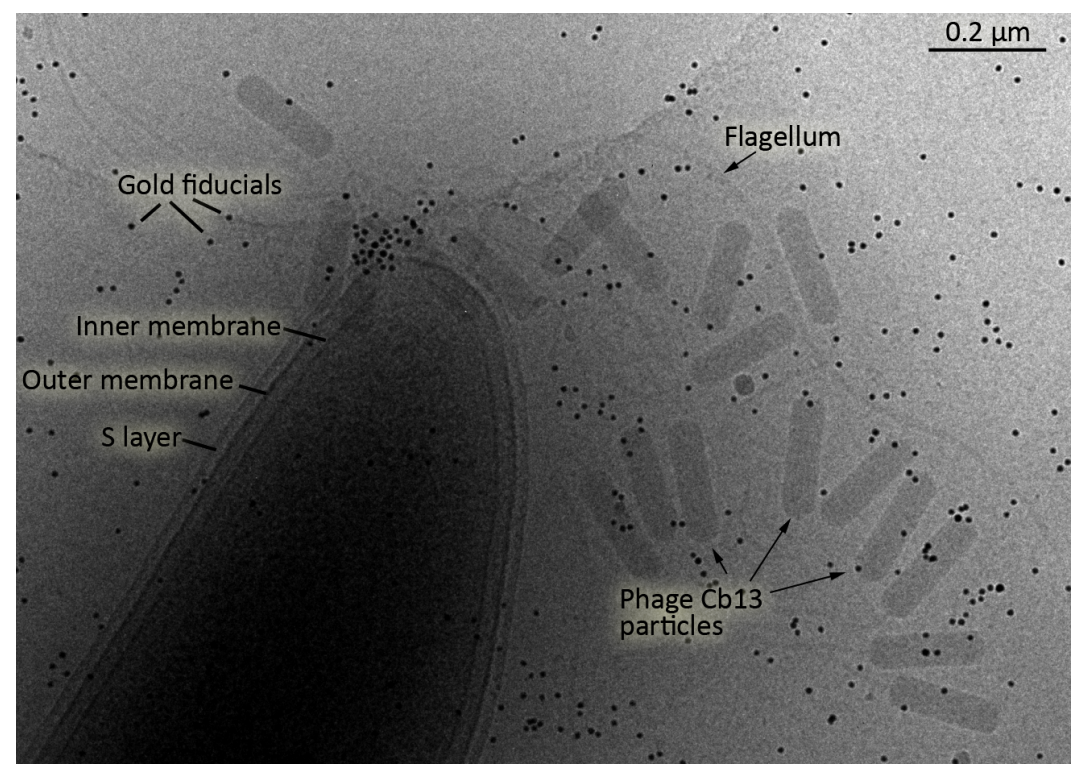

FIG. 1. Cryo-electron micrograph of plunge-frozen C. crescentus $\Delta m o t A$ strain infected with phage $\varphi \mathrm{Cb} 13$. Notice that flagellum-associated phage particles are oriented with their heads towards the flagellum. Phages attached to the cell pole are also observed.

TABLE 2. Motility assays and rate of phage adsorption to wild type and motility mutants.

\begin{tabular}{|c|c|c|c|c|c|c|c|}
\hline \multirow{2}{*}{ Strain name } & \multirow{2}{*}{$\begin{array}{l}\text { Motility } \\
\text { zone } \\
\text { diameter } \\
\text { (48 hours) }\end{array}$} & \multicolumn{3}{|c|}{$\begin{array}{c}\varphi \text { Cb13 Adsorption } \\
\left(\mathrm{K} \text { values }=\times 10^{-11} \mathrm{~mL} / \mathrm{min}\right)\end{array}$} & \multicolumn{3}{|c|}{$\begin{array}{c}\varphi \text { CbK Adsorption } \\
\text { (K values }=\times 10^{-11} \mathrm{~mL} / \mathrm{min} \text { ) }\end{array}$} \\
\hline & & Slope & r value & $\mathrm{k}$ value & Slope & $\mathrm{r}$ value & $\mathrm{k}$ value \\
\hline C. crescentus NA1000 & $6 \mathrm{~mm}$ & -0.0150 & 0.9888 & 3.71 & -0.0157 & 0.9955 & 3.49 \\
\hline C. crescentus motA:Tn & $0 \mathrm{~mm}$ & -0.0036 & 0.9073 & 0.60 & -0.0073 & 0.9975 & 1.62 \\
\hline C. crescentus cheB144::Tn & $2 \mathrm{~mm}$ & -0.0090 & 0.9960 & 2.40 & -0.0092 & 0.9672 & 2.04 \\
\hline C. crescentus cheB148::Th & $2 \mathrm{~mm}$ & -0.0011 & 0.9753 & 2.78 & -0.0105 & 0.9678 & 2.33 \\
\hline C. crescentus cheR::Tn (NS209) & $4 \mathrm{~mm}$ & -0.0043 & 0.9127 & 0.62 & -0.0058 & 0.9947 & 1.29 \\
\hline C. crescentus cheR::Tn (NS338) & $4 \mathrm{~mm}$ & -0.0047 & 0.9385 & 0.71 & -0.0079 & 0.9965 & 1.76 \\
\hline C. crescentus $\Delta$ tipF & $0 \mathrm{~mm}$ & -0.0023 & 0.9256 & 0.67 & -0.0083 & 0.9938 & 1.84 \\
\hline C. crescentus $\triangle$ pilA & $7 \mathrm{~mm}$ & -0.0007 & 0.6481 & 0.13 & -0.0027 & 0.9521 & 0.60 \\
\hline
\end{tabular}

